\title{
Review of Noise Filtering Algorithm For Photon Data
}

Huang jiapeng Xing yanqiu Qin lei

College of Engineering and Technology, Northeast Forestry University, Harbin, China, 150000

KEY WORDS:ICESat-2/ATLAS, photon data, simulated photon-counting lidar, noise filtering algorithm

\section{ABSTRACT:}

As a continuation of Ice, Cloud, and Land Elevation Satellite-1 (ICESat-1)/Geoscience Laser Altimeter System (GLAS), the Ice, Cloud, and Land Elevation Satellite-2 (ICESat-2), which is equipped with the Advanced Topographic Laser Altimeter (ATLAS) system, was successfully launched in 2018. Since ICESat-1/GLAS has facilitated scientific results in the field of forest structure parameter estimation, how to use the ICESat-2/ATLAS photon cloud data to estimate forest structure parameters has become a hotspot in the field of spaceborne photon data application. However, due to the weak photon characteristics of the ICESat-2/ATLAS system, the system is extremely susceptible to noise, which poses a challenge for its subsequent accurate estimation of forest structural parameters. Aiming to filter out the noise photons, the paper introduces the advantages of the spaceborne lidar system ICESat-2/ATLAS than ICESat-1/GLAS. The paper summarizes the research of the simulated photon-counting lidar (PCL) noise filtering algorithm and noise filtering on spaceborne.

\section{INTRODUCTION}

Forest structural parameters have been listed as one of the important indicators for monitoring forest carbon by the International Union of Forest Research Organizations (IUFRO). How to quickly and accurately quantificate the forest structure parameters of the study area is great significance for monitoring forest carbon (Jandl, 2019 and Golshani, 2019). In the process of forest monitoring, forest structural parameters (such as canopy height, et al.) directly reflect the growth of forests, and have become one of the most commonly used bases in forest ecological research (Wu, 2019). Traditional forest monitoring methods are mostly acquired by manual field measurement. Although the measurement is accuracy, it has a long working cycle and low efficiency and not applicable complete large-scale, multi-scale for forest structure parameter monitoring (Piermattei, 2019) .

In recent years, with the development of remote sensing technology, remote sensing technology has become a key technology for monitoring carbon, and has been identified as a monitoring method that complies with the "Kyoto Protocol" (Lefsky, 2002). Different from optical remote sensing image (Mohd, 2018) and synthetic aperture radar (SAR) (Zhang, 2019) by cloud and biomass saturation, spaceborne Light Detection And Ranging (LiDAR) technology overcomes the above problem from the perspective of data source, and has been widely used with its efficient and accurate ranging capability. In order to acquire large-area, multi-scale, multi-temporal forest monitoring data, studies used the spaceborne lidar data to retrieve forest structural parameters. So far, two spaceborne lidar could provide scientific data, include Ice, Cloud, and Land Elevation Satellite (ICESat-1) launched by the NASA in 2003, (Wang, 2019, Hu, 2019 and Lefsky, 2010), and Ice, Cloud, and Land Elevation Satellite (ICESat-2) (Neuenschwander, 2019) successfully launched in 2018 , respectively. ICESat-2 is a continuation of the ICESat-1 scientific mission, planning to provide scientific and accurate photon data for global forest biomass (Sawruk, 2018 and Markus, 2017). However, because the ICESat-2 system has the characteristic of photon-counting, the signal photons emitted and received are weak signal photons, which are greatly affected by background noise (solar background noise, system dark noise, et al.). Therefore, how to effectively filiter noise photon is the hotspot in photon data processing and application ( $\mathrm{Li}, 2018$, Huang, 2019 and Moussavi, 2014).

In order to advance the progress of the spaceborne photon noise filitering algorithm, this paper will introduce the advantages of the spaceborne lidar system ICESat-2 and its relative ICESat-1/GLAS, and 
summarize the simulated photon data noise filitering algorithm. The research prospect the trend of photon data noise filitering algorithm.

\section{MAIN BODY}

\subsection{ICESat-2/ATLAS and ICESat-1/GLAS}

ICESat-2 was successfully launched on September 15, 2018. The system is equipped with Advanced Topographic Laser Altimeter (ATLAS). The system adopt photon-counting technology, which has the characteristics of high repetitive frequency and low energy (Moussavi, 2014), the laser emission mode of the system is multi-beam emission mode, the emission mode is 3 pairs laser beam and in each pair of beams with the ratio of $4: 1$. This multi-beam laser emission method can increase the observation data of the same route, and can provide more information for the slope and vegetation coverage of the study area. (Molly, 2019 and Montesano, 2015). The laser emission frequency is $10 \mathrm{kHz}$. Combined with the satellite orbital altitude and satellite operating speed, the footprint size is $17 \mathrm{~m}$ (nominal) and the footprint spacing is $0.7 \mathrm{~m}$. This high-frequency transmission could obtain approximate continuous information, and since ATLAS has a smaller footprint diameter than the Geoscience Laser Altimeter System (GLAS), the small footprint can more clearly describe surface features than the footprint. The small footprints are less affected by the terrain, and improving the accuracy of the measurement. These features will continue the ICESat-1/GLAS mission, providing more accurate and scientific data for global canopy height (Kelly, 2019, Christopher, 2019 and Lana, 2019).

Limited to the hardware characteristics of the ICESat-1/ATLAS system, the laser emission mode of the system is single beam transmission mode, the laser emission frequency is $40 \mathrm{~Hz}$, combined with the satellite orbital height and the satellite running speed, the footprint size is $66 \mathrm{~m}$. The spacing of $170 \mathrm{~m}$ along the track makes the GLAS system unable to obtain continuous information. Because of the large footprint, ICESat-1/GLAS contains more ground information, which is more susceptible to information such as terrain slope (Yang,2019, Moussavi,2014 and
Yang,2019). Unlike the waveform technology used in the ICESat-1/GLAS system, the ICESat-2/ATLAS system will use photon-counting technology. The GLAS laser has high pulse energy, low frequency, and measured target information by waveform. The ICESat-2/ATLAS has low pulse energy and high frequency. The recorded target information is determined by recording the returned photon time tag information. The ICESat-2/ATLAS system has three advantages over the ICESat-1/GLAS system. Firstly, its photon density is denser than the ICESat-1/GLAS system (ATLAS is $0.7 \mathrm{~m}$ along the foot footprint, GLAS is $170 \mathrm{~m}$ along the foot footprint), could acquire continuous information, and the information of the measured target can be more accurately described, which is significance for improving the extraction of forest structure parameters. Secondly, the ICESat-2/ ATLAS system is a multi-beam transmission method, and could acquire more observation information under the same orbit. Thirdly, the ICESat-2/ATLAS system has a smaller footprint diameter (17m for ATLAS and $70 \mathrm{~m}$ for GLAS), which reduces the impact of slope on spot information acquisition.

2.2 Review of Simulated Photon LiDAR Noise Filitering Algorithm

In order to simulate the ICESat-2/ATLAS spaceborne photon data noise filitering algorithm, the noise filitering algorithm concentrated on single photon radar (SPL) and Multiple Altimeter Beam Experimental LiDAR (MABEL), Slope Imaging Multi-Polarization Photon Counting Lidar (SIMPL), MATLAS data. Xia et al (2014) and Xu. et al (2014, 2017) used the MABEL data under night conditions as the experimental data. The algorithm firstly projects the photon data to the local coordinate system and converts the plane coordinates of the photon data into the orbital distance, thus generating a 2-dimensional elevation photon data profile. Then calculating the total distance of each photon to the $\mathrm{k}^{\text {th }}$-nearest photons and statistics the frequency histogram of the local total distance. Finally, the noise photon is removed by setting the threshold. The noise filitering result indicates that the algorithm can effectively eliminate most of the noise photons under nighttime observation 
conditions. However, the study data is nighttime photon data, which is less affected by solar background noise, so noise filitering can filiter noise photon data by threshold statistics.

Wang et al. (2016) used MABEL data as the research data, and proposed the probability distribution function of k-th nearest neighbors $(\mathrm{KNN})$ to judge the distance of each photon cloud, and then applied Bayesian probability theory to calculate signal photons. The noise filitering results show that the algorithm can effectively distinguish between noise photons and signal photons. However, the noise photons distribution are relatively random and are closely related to the observation target. It is difficult to accurately determine the photon property only by the probability of detection.

Yu. (2016) used the photon-counting laser data of the laboratory platform as the research data to analysis the noise filitering performce of the moving surface fitting method and the mathematical morphology filtering algorithm. The processing targets are hypothetical targets. Combining the advantages and disadvantages of the two methods, an improved noise filitering algorithm is proposed based on improved mathematical surface morphology. The experimental results show that the noise filitering algorithm can filiter noise photon data in the laboratory platform, but manually setting the threshold is the key to noise filitering algorithm.

Magruder et al. (2012) proposed three methods for noise filitering photon data, such as Canny Edge Detection (CED) algorithm, Probability Distribution Function (PDF) and local angle mapping method. The result proves that the CED algorithm in the forest research area has better noise filitering ability, but the signal photon loss event is prone to occur in the gridding process, which will reduce the effectiveness of noise filitering.

Tang et al. (2016) proposed a new voxel-based spatial filtering method based on SPL data as experimental data, and determined the optimal voxel size for the optimal filter by testing different voxel sizes. It is finally determined that Garrett algorithm is the optimal algorithm for the experimental data, and
$3 \mathrm{~m} \times 3 \mathrm{~m} \times 0.2 \mathrm{~m}$ is the optimal voxel size of the test data. This method effectively filters the noise photon of the smooth surface while maintaining the spatial integrity of the SPL data, but the algorithm has filitered less photon in the research area with forest cover on the surface, and the SPL data is high density photon cloud data. There is a significant difference between the SPL data and the ATLAS photon data, so the voxel-based spatial filtering algorithm is not necessarily applicable in the ATLAS photon data.

Nie et al. (2018) proposed a local statistical analysis algorithm based on strong beam laser type MATLAS data as experimental data. The algorithm establishes the frequency histogram based on the elevation of all photon data. Then uses the waveform analysis method to identify the peak location of the frequency histogram, set a reasonable elevation threshold range near the peak location. Then, the statistics and normalizate the photon density. Finally, the local distance statistical algorithm is used to filiter noise photons. Experiment results show that the algorithm can effectively remove noise photons in the photon data. However, this type of algorithm needs to manually change the threshold parameters of local statistics according to different study area, which reduces the applicability of the noise filitering algorithm.

Zhu et al. (2018) proposed an improved elevation frequency histogram noise filitering algorithm. Firstly, the method calculates the photon search region in a horizontal elliptical shape, density of each photon and the maximum of each photon. Then, algorithm generates the local photon density distribution histogram by the calculated photon density. Since the signal photon density is much higher than the noise photon density, the low-density photon can be regarded as a noise photon. The photon density threshold is set by fitting the noise peak and the signal peak using Gaussian curve to classify the original photon into signal photons and noise photons.

Based on the different characteristics of noise photon density and signal photon density, scholars have chosen different density spatial clustering algorithms as noise filitering algorithms into photon data. Among 
them, Herzfeld et al. (2014) used statistical classification parameters and feature type hyper-parameters as auxiliary parameters, and proposed a photon denoising algorithm using spatial statistics and discrete mathematical concepts. The experimental results show that the algorithm can effectively identificate the signal photons in the photon data and provide the data basis for photon feature classification. Herzfeld et al. (2017) proposed a Density Dimension Algorithm (DDA), which calculates the Radial Basis Function (RBF) under anisotropic conditions. The weighted density of the data aggregation form and the concept of considering the density additional dimension is introduced as the auxiliary of the adaptive threshold determination. The photon noise filitering experiment is completed by the low pass filter. The results show that the algorithm has better adaptive ability. It can effectively distinguish noise photons and signal photons. However the algorithm does not have the ability to effectively identify signal photons with forest cover complex terrain.

Popescu et al. (2018) used MABEL data as experimental data to filier noise based on integrated moving window and clustering algorithm. Firstly, the terrain reference range is defined by the auxiliary digital elevation model (DEM), the noise photons far away from the surface are removed by the hard threshold method, and then the noise photon is filiering by the clustering algorithm with $\log$ likelihood function. Finally, the residual abnormal noise photons are removed by a Confidence Interval (CI) filter. The results show that the algorithm can identify the background noise in the flat study area and preserve the signal photons in the original data, however, the algorithm does not have the ability to effectively identify signal photons with forest cover complex terrain.

Due to the ICESat-2/ATLAS photon density characteristics, the photon density in the horizontal direction is higher than the vertical direction. Therefore, Zhang et al. (2014) proposed an improved density spatial clustering algorithm modified Density Based Spatial clustering of Applieations with Noise
(mDBSCAN), for each photon object in the same cluster, the number of samples contained in the neighborhood Eps of a given radius cannot be less than a certain threshold MinPts as the cluster basis. Based on the clustering of photon objects with density connection properties. In the original photon data set, clustering can continue as long as the density of photon data exceeds MinPts. These photon objects are identified as signal photons, otherwise, these photons are identified as noise photons. The algorithm uses the horizontal elliptical shape to calculate the distance between photons, which makes the algorithm have better noise filitering effect in the flat test area. On the basis of this research, Chen et al. (2019) proposed the distance between photons by using vertical elliptical shape, horizontal elliptical shape and circular shape. The algorithm is completed under the condition of strong beam laser type. The results show that the horizontal ellipse search domain is more suitable for the flat photon cloud noise filitering algorithm.

\section{CONCLUSIONS}

According to the research, it is recommended to pay attention to the following problems in the future study: 1) The photon noise filtering algorithm has high precision on smooth surfaces, while in the forest coverage area, due to noise photons distributed over the canopy, inside the canopy and below the ground, which seriously affects the accuracy of the noise filtering algorithm. 2) The above research only selects one type of airborne route. The simulated PCL data is not analysis noise filtering by laser pointing and strong and weak type, lacking comprehensive analysis and evaluation of noise filtering effect. 3) Most algorithms need to manually adjust the parameter of the noise filtering algorithm. It also limits the adaptability of the algorithm. Therefore, how to improve the noise filtering effect of the spaceborne photon data in the forest coverage area by an adaptive way is the technical difficulty in photon data processing.

\section{REFERENCES}

Jandl, R., Spathelf, P., Bolte, A., Prescott, C. E. , 2019

Forest adaptation to climate change-is non- 
management an option? Annals of Forest Science, 76(2).1-13.

Golshani, P., Maghsoudi, Y., Sohrabi, H., 2019: Relating ALOS-2 PALSAR-2 Parameters to Biomass and Structure of Temperate Broadleaf Hyrcanian Forests. Journal of the Indian Society of Remote Sensing.

Wu, L., Zhu, X., Lawes, R., Dunkerley, D., Zhang, H., 2019: Comparison of machine learning algorithms for classification of LiDAR points for characterization of canola canopy structure. International Journal of Remote Sensing, 1-19.

Piermattei, L., Karel, W., Wang, D., Wieser, M., Mokroš, M., Surový, P., Hollaus, M.,2019: Terrestrial Structure from Motion Photogrammetry for Deriving Forest Inventory Data. Remote Sensing, 11(8), 950. Lefsky, M. A., Cohen, W. B., Harding, D. J., Parker, G. G., Acker, S. A., Gower, S. T., 2002: Lidar remote sensing of above-ground biomass in three biomes. Global Ecology and Biogeography, 11(5), 393-399.

Najib, N. E., Kanniah, K. D., 2018: Optical and radar remote sensing data for forest cover mapping in Peninsular Malaysia. Singapore Journal of Tropical Geography, 1-19.

Zhang, Y., Ling, F., Foody, G. M., Ge, Y., Boyd, D. S., Li, X., Atkinson, P. M., 2019: Mapping annual forest cover by fusing PALSAR/PALSAR-2 and MODIS NDVI during 2007-2016. Remote Sensing of Environment, 224, 74-91.

Wang, Y., Ni, W., Sun, G., 2019: Slope-adaptive waveform metrics of large footprint lidar for estimation of forest aboveground biomass. Remote Sensing of Environment, 224: 386-400.

Hu, Y., Wu, F., Sun, Z., Lister, A., Gao, X., Li, W., Peng, D., 2019: The Laser Vegetation Detecting Sensor: A Full Waveform, Large-Footprint, Airborne Laser Altimeter for Monitoring Forest Resources. Sensors, 19(7), 1699.

Lefsky, M. A., Harding, D. J., Keller M., et al, 2005: Estimates of forest canopy height and aboveground biomass using ICESat. Geophysical Research Letters, 32(22):L22S02.
Neuenschwander, A., Pitts K.,2019: The ATL08 land and vegetation product for the ICESat-2 Mission. Remote Sensing of Environment, 221, 247-259.

Sawruk, N., Burns P., Edwards, R., Litvinovitch, V., Hovis, F., 2018: Flight Lasers Transmitter Development for Nasa Ice Topography Icesat-2 Space Mission. IGARSS 2018-2018 IEEE International Geoscience and Remote Sensing Symposium.

Markus, T. , Neumann, T. , Martino, A. , et al., 2017: The Ice, Cloud, and land Elevation Satellite-2 (ICESat-2): Science requirements, concept, and implementation. Remote Sensing of Environment, 190 : 260-273.

Li, G., Huang, J., Tang, X., et al., 2018: Influence of Range Gate Width on Detection Probability and Ranging Accuracy of Single Photon Laser Altimetry Satellite. Acta Geodaetica et Cartographica Sinic, 47 (11):1487-1494.

Huang, J., Xing, Y., You, H., Qin, L., Tian, J.,Ma, J. , 2019: Particle Swarm Optimization-Based Noise Filtering Algorithm for Photon Cloud Data in Forest Area. Remote Sensing, 11(8), 980.

Yang, X., Wang, C., Pan, F., Nie, S., Xi, X., Luo, S., 2019: Retrieving leaf area index in discontinuous forest using ICESat/GLAS full-waveform data based on gap fraction model. ISPRS Journal of Photogrammetry and Remote Sensing, 148, 54-62.

Moussavi, M. S., Abdalati, W., Scambos, T., et al., 2014: Applicability of an automatic surface detection approach to micro-pulse photon-counting lidar altimetry data: implications for canopy height retrieval from future ICESat-2 data. International Journal of Remote Sensing, 35(13):5263-5279.

Molly, E.,Vanessa, M., 2019: NASA's Early Adopter Program Links Satellite Data to Decision Making. Remote Sensing, 11, 406.

Montesano, P. M., Rosette, J., Sun, G., et al., 2015: The uncertainty of biomass estimates from modeled ICESat-2 returns across a boreal forest gradient. Remote Sensing of Environment, 158:95-109.

Kelly, M., Thomas, A., Christopher, F., 2019: Assessment of altimetry using ground-based GPS data from the 88S Traverse, Antarctica, in support of ICESat-2.The Cryosphere, 13, 579- 590. 
Christopher, J., Jeannette, v., Kelly, M., 2019: Radiometric calibration of a non-imaging airborne spectrometer to measure the Greenland ice sheet surface.Atmos. Meas. Tech, 12, 1913-1933.

Lana, L., Sorin, P., Amy, N., 2019: Estimating aboveground biomass and forest canopy cover with simulated ICESat-2 datat. Remote Sensing of Environment, 224, 1-11.

Xia, S., Wang, C., Xi, X., 2014: Point cloud filtering and tree height estimation using airborne experiment data of ICESat-2. Journal of Remote Sensing, 18:1199-1207.

Xu, Y., Li, G., Qiu, C., Xu, Y., 2019: Single photon laser data processing technology based on Terrain Correlation and least square curve fitting.Infrared and Laser Engineering, 1-10.

Xu, Y., 2017: Research on Data Processing Technology of Single Photon Laser Alimetry. Xi'an: Xi'an University of Science and Technology.

Wang, X., Pan, Z., 2016: Glennie,Craig.A Novel Noise Filtering Model for Photon-Counting Laser Altimeter Data. IEEE Geoscience \& Remote Sensing Letters, 13:947-951.

Yu, F., 2016: Research on laser 3D point cloud data processing method based on single photon detection. Harbin: Harbin Institute of Technology.

Magruder, L.A., Neuenschwander, A.L., 2012: Noise filtering techniques for photon-counting ladar data. Proceedings of SPIE - The International Society for Optical Engineering, 8379, 1-9.

Tang, H., Swatantran, A., Barrett,T., et al. 2016: Voxel-Based Spatial Filtering Method for Canopy Height Retrieval from Airborne Single-Photon Lidar.Remote Sensing, 8, 1-13.

Nie, S.,Wang, C., Xi,X., et al, 2018: Estimating the vegetation canopy height using micro-pulse photon counting LiDAR data. Optics Express, 26: 520-540.

Zhu, X., Nie, S., Wang, C., Xi, X., Hu, Z., 2018: A Ground Elevation and Vegetation Height Retrieval Algorithm Using Micro-Pulse Photon-Counting Lidar Data. Remote Sensing, 10(12), 1962.

Herzfeld, U.C., Trantow, T.M., Harding, D., et al. 2017: Surface Height Determination of Crevassed Glaciers Mathematical Principles of an Autoadaptive
Density Dimension Algorithm and Validation Using ICESat-2 Simulator (SIMPL) Data.IEEE Transactions on Geoscience \& Remote Sensing, 99:1-23.

Herzfeld, U.C., Mcdonald, B.W., Wallin, B.F., et al. 2014: Algorithm for Detection of Ground and Canopy Cover in Micropulse Photon-Counting Lidar Altimeter Data in Preparation for the ICESat-2 Mission. IEEE Transactions on Geoscience \& Remote Sensing, 52: 2109-2125.

Popescu, S. C., Zhou, T., Nelson, R., et al, 2018: Photon counting LiDAR: An adaptive ground and canopy height retrieval algorithm for ICESat-2 data.Remote Sensing of Environment, 208:154-170.

Zhang, J., Kerekes, J., 2014: An Adaptive Density Based Model for Extracting Surface Returns From Photon-Counting Laser Altimeter Data. IEEE Geoscience \& Remote Sensing Letters, 12: 726-730.

Chen B., Pang Y., Li Z., 2019: Ground and Top of Canopy Extraction from Photon Counting LiDAR Data Using Local Outlier Factorwith Ellipse Searching Area. IEEE GEOSCIENCE AND REMOTE SENSING LETTERS. 